\title{
Wide bandwidth, millimeter-resolution inverse synthetic aperture radar imaging
}

\author{
Jonathan T. Richard, ${ }^{1}$ Martin S. Heimbeck, ${ }^{2}$ L. Blake Autin, ${ }^{1}$ and Henry O. Everitt ${ }^{2,3, *}$ \\ ${ }^{1}$ IERUS Technologies, Huntsville, Alabama 35805, USA \\ ${ }^{2}$ Charles M. Bowden Research Center, Army Aviation \& Missile RD\&E Center, Redstone Arsenal, Huntsville, Alabama 35898, USA \\ ${ }^{3}$ Department of Physics, Duke University, Durham, North Carolina 27708, USA \\ *Corresponding author: everitt@phy.duke.edu
}

Received 9 December 2016; revised 12 May 2017; accepted 12 May 2017; posted 15 May 2017 (Doc. ID 282562); published 6 June 2017

\begin{abstract}
The combination of wide bandwidth W-band inverse synthetic aperture radar imagery and high-fidelity numerical simulations has been used to identify distinguishing signatures from simple metallic and dielectric targets. Targets are located with millimeter-scale accuracy using super-resolution techniques. Radon transform reconstructions of the returns from rotated targets approached the image quality of the complete data set in a fraction of the time by sampling as few as 10 angles. The limitations of shooting-and-bouncing ray simulations at high frequencies are illustrated through a critical comparison of their predictions with the measured data and the method of moments simulations, indicating the importance of accurately capturing the obfuscating role played by multipath interference in complex targets. () 2017 Optical Society of America
\end{abstract}

OCIS codes: (100.0100) Image processing; (100.2960) Image analysis; (100.6640) Superresolution; (110.0110) Imaging systems; (110.6795) Terahertz imaging; (280.6730) Synthetic aperture radar.

https://doi.org/10.1364/JOSAA.34.001073

\section{INTRODUCTION}

The use of advanced radar imaging techniques, such as synthetic aperture radar (SAR) and inverse synthetic aperture radar (ISAR), is being adapted from the radio frequency (RF) and microwave (MW) into the millimeter-wave (mmw) and terahertz $(\mathrm{THz})$ spectral regions [1-10]. The high-fidelity images produced by these techniques at high frequencies have vindicated the high expectations for applications ranging from non-destructive testing and seeing through obscurants to terrain mapping and navigation assistance. High-frequency radar demonstrations have typically used impressive proof-of-concept images to illustrate the successful construction of the necessary imaging technology. However, for these techniques to demonstrate their full potential for thorough analysis of complex scenes, such imagery must be coupled with appropriate simulations so that the scene may be discriminately interpreted and artifacts identified. For radar simulations, point-scatter models have been derived from measurement data but were not modeled in simulations $[11,12]$. The most widely used modeling simulations for this type of imagery use physical optics and the shooting-and-bouncing ray (SBR) approximations, but at high frequencies these approximations fail to produce accurate reconstructions with noticeable limitations, particularly in cross-polarization $[6,13]$. More rigorous simulations, such as the method of moments (MoM), produce much more accurate and reliable analyses, but as the wavelength decreases in comparison to the size of the target objects, these techniques become computationally intensive to the point of impracticality because of hardware limitations, even when approximations such as the asymptotic waveform evaluation technique are used [14-16]. While these measurements and simulations have been separately performed and images have been generated, model/measurement agreement is often difficult to achieve with confidence due to decreased stability and registration tolerances in the experiments, the increased material-dependent complexity of simulations, and the limitations of computational hardware.

Here we present W-band ISAR images of several simple metallic and dielectric targets, reconstructed through the use of Radon transforms, and critically compare them with predicted images using SBR and MoM simulations. Distinguishing signatures of metallic and dielectric scatterers are identified to provide a foundational approach to correlating measured and simulated data for high-frequency, high-bandwidth, and high-resolution imagery. The comparison of SBR and MoM simulations with the measured data indicates unphysical artifacts, inadequate predictions of multipath interference, and inferior reconstruction fidelity of the former, an anticipated result given that SBR techniques are increasingly inaccurate at higher frequencies. Nevertheless, the data and simulations reveal clear signatures that distinguish metallic from dielectric scatterers and capture the effects of multipath interference, while super-resolution 
(SR) techniques were able to achieve reconstructions with millimeter-scale accuracy. Given that the speed of image acquisition depends on the number of angular samplings taken, the quality of the image degradation is measured as a function of the number of samplings, and it is found that the targets are accurately located in fewer than 10 samples. These analyses indicate the need for high fidelity simulation tools to help interpret such high-resolution data and provide a methodology for ascertaining the minimum number of samples required to obtain such image reconstructions rapidly using commercially available technology.

\section{METAL AND DIELECTRIC RESPONSES}

\section{A. Methods}

The experimental system shown in Fig. 1 was built using an Agilent N5222A vector network analyzer (VNA), W-band transceiver, focal mirror, a Newport rotational stage, and a computer to control the hardware. A Virginia Diodes (VDI) WR10 transceiver was used to transmit and receive W-band data. Radiation was coupled out of the W-band transceiver by a standard gain horn antenna and was collimated by a focal mirror producing a Gaussian beam waist of $\sim 15 \mathrm{~cm}$ at the target. Calibration of the VNA removed undesired stationary reflections that were consistently seen by the radar. The VNA was swept over the entire W-band $(75-110 \mathrm{GHz})$ with an intermediate frequency (IF) bandwidth of $100 \mathrm{~Hz}$ to minimize measurement noise. Typical signal-to-noise ratios ranged from $30-45 \mathrm{~dB}$ with a noise floor near $-60 \mathrm{dBm}$. The data was analyzed using a Hamming window [17] followed by a MATLAB algorithm to compute an inverse fast Fourier transform so that the range domain may be calculated from the frequency data with a range resolution $\Delta r$ of

$$
\Delta r=\frac{c}{2 B},
$$

for frequency bandwidth $B$ and the speed of light $c$. For this experiment, the range resolution achieved with a bandwidth of $B=35 \mathrm{GHz}$ is $\Delta r=4.3 \mathrm{~mm}$.

The first experiment was to characterize and compare the ISAR signatures of metallic and dielectric rods of the same diameter located at the center of rotation. The metallic target was an aluminum rod, treated as a perfect electrical conductor (PEC), with a diameter of $6.4 \mathrm{~mm}$ and a height of $304.8 \mathrm{~mm}$. The dielectric target was a Teflon rod with the same dimensions as the metallic rod and a relative permittivity of 2. Next, a more complex target was constructed (Fig. 1), composed of two aluminum rods each with a diameter of $6.4 \mathrm{~mm}$, one dielectric rod with a diameter of $6.4 \mathrm{~mm}$, and one rectangular $25.4 \times 12.7 \mathrm{~mm}$ aluminum block, all $304.8 \mathrm{~mm}$ tall and mounted on a rotational stage. The rod located at the center of rotation was either an aluminum or Teflon rod, and the locations of the rods were measured with a ruler to a respective absolute radial and angular accuracy of $\pm 1 \mathrm{~mm}$ and $\pm 1^{\circ}$ for comparison with the locations extracted from ISAR measurements without and with the use of a SR algorithm. Measurements were captured by rotating the target in $1^{\circ}$ increments through $360^{\circ}$. The lengths of the rods and blocks were parallel to the polarization (linearly vertically polarized).

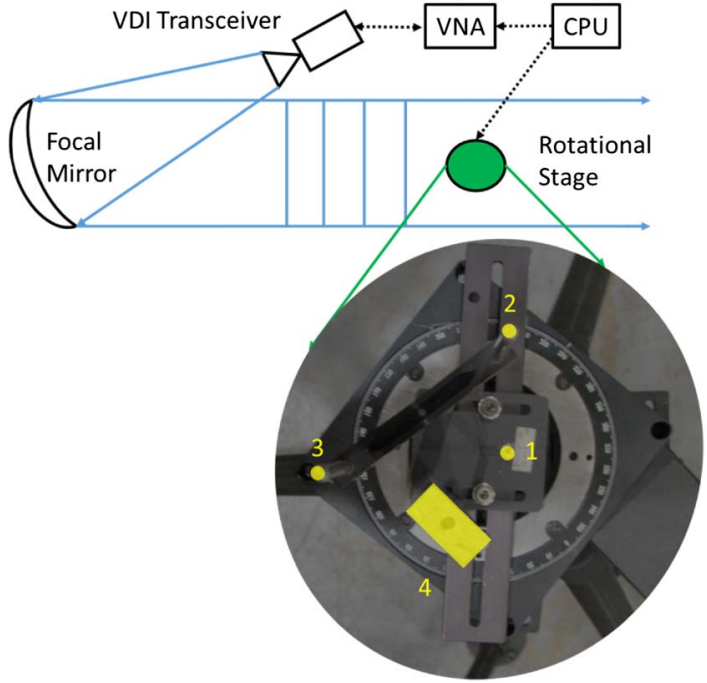

Fig. 1. Top view of the ISAR experimental setup, illustrating the complex target composed of one rod at the center of rotation (1), two PEC rods $(2,3)$, and one PEC rectangle (4). The rod at the center of rotation was either PEC or dielectric.

Two techniques are used to simulate the ISAR data: the MoM technique V-Lox and the shooting and bouncing ray technique XPATCH $[18,19]$. V-Lox is a high-performance computational electromagnetic simulation software suite based upon the MoM technique that implements a surface integral equation formulation on a triangular mesh [20]. The MoM procedure approximates the integral equations with a matrix of linear algebraic equations to model electrical current distributions on a body's surface, from which the radiation pattern and impedances may be calculated. The matrix is composed of $N$ equations and $N$ unknowns to represent a mesh of the surface being modeled, so the matrix size increases by the square of the number of unknowns that are being solved [21]. V-Lox is intended to solve problems involving electrically small to large objects made up of a mixture of PEC, dielectric, and magnetic materials, and it supports a fast direct-solve adaptive cross approximation technique for electrically large problems, which includes a multigraphics processor unit (GPU) accelerated block lower upper (LU) decomposition algorithm [22]. Additional computational gain in rigorous MoM modeling was accomplished through the use of GPU acceleration, advances in random access memory (RAM), and solid-state drive flash storage. V-Lox computations presented here typically required an hour on a PC using an Intel Core-I7 and 16 GB RAM.

As implemented in XPATCH, the SBR technique is able to generate radar cross sections, range domain signatures, and SAR images of realistic objects [19] using a computer-aided design (CAD) methodology to simulate the target structure, collecting multibounce ray contributions from these complex objects and materials [18]. Comparisons between simulation and measurement have been performed from UHF through Ka-band frequencies, but SBR's appropriateness for frequencies above Ka-band has been questioned because a significant body of verified data does not exist to warrant a high confidence level in the 
results [23]. In the simulations used here, we used the standard assumption that, for a given look angle, a target whose physical cross section is $\mathrm{m} \times \mathrm{n}$ wavelengths is well characterized by $10 \mathrm{~m} \times 10 \mathrm{n}$ rays. XPATCH computations presented here typically required a few minutes on the same PC used for V-Lox.

\section{B. Comparison of Metallic and Dielectric Rods}

Figure 2(a) compares the measured return from the metallic and dielectric rods located at the center of rotation. To calibrate the experiment and define the center of rotation as $0 \mathrm{~mm}$ down range, we assumed that the spatial location of the peak signal from the PEC rod was produced by its front surface reflection. This occurred at a down-range distance equal to the rod's radius of $-3.2 \mathrm{~mm}$, where the negative sign indicates the front surface is closer to the source. Range-aspect-intensity (RAI) plots, shown in Figs. 2(b), 2(c), 2(e), and 2(f), summarize downrange measurements as a function of aspect angle. Figure 2(b)
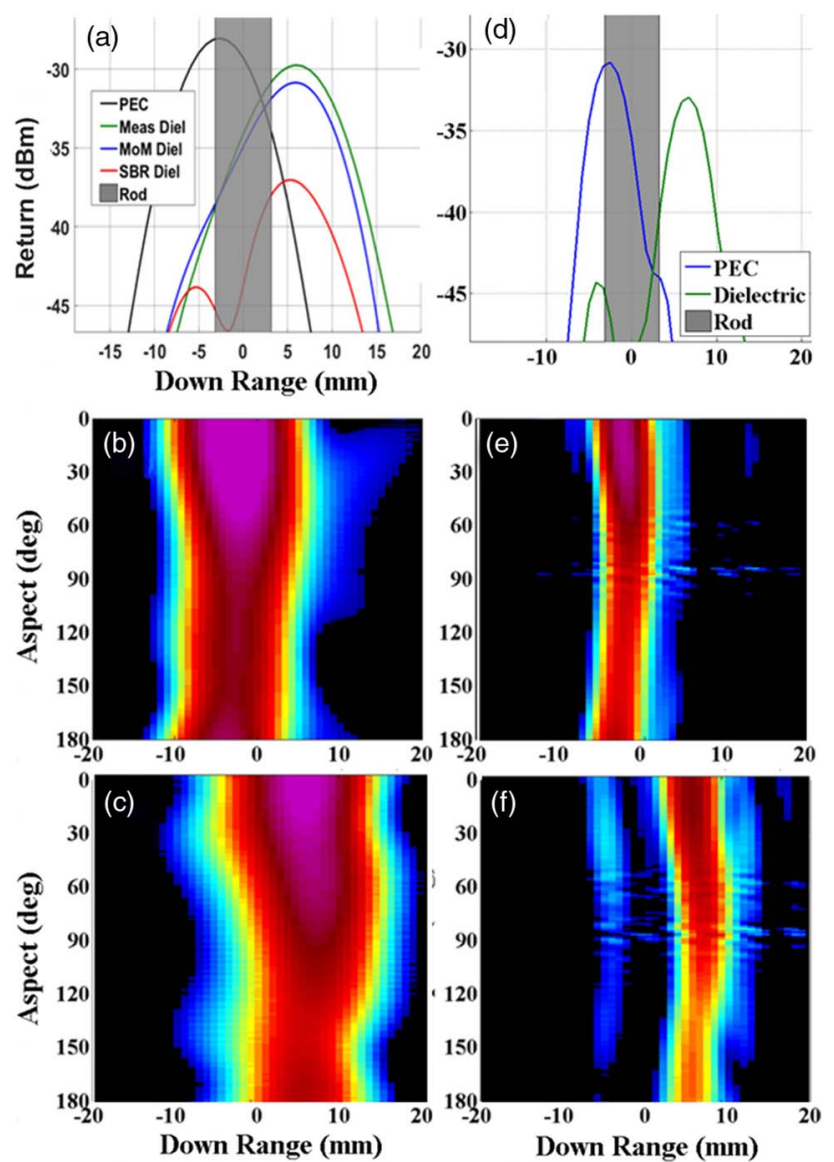

Fig. 2. (a) Measured down-range returns for a $75-110 \mathrm{GHz}$ frequency sweep from the PEC and dielectric rods at the same physical location, showing a $9 \mathrm{~mm}$ range delay from the front surface for the latter, and the predicted dielectric rod down-range returns calculated from MoM and SBR simulations with a $6.4 \mathrm{~mm}$ diameter rod $\left(\epsilon_{r}=2\right)$. The corresponding RAI of measurements from (b) a PEC rod and (c) a dielectric rod are plotted as a function of rotation angle. (d) Measured down-range returns of the PEC and dielectric rods using SR, showing both the front return and the $9 \mathrm{~mm}$ range delayed return for the dielectric. The corresponding SR RAI of (e) a PEC rod and (f) a dielectric rod plotted as a function of rotation angle. The dynamic range from purple to black spans $45 \mathrm{~dB}$ in the RAI plots. shows that the range domain return remains aligned with the front of the $6.4 \mathrm{~mm}$ diameter PEC rod for all aspect angles. The range resolution estimated above is consistent with the width of the measured return, and the slight angular variations in the peak return are a consequence of an imperfect vertical alignment of the rod.

The same offset was used to align the dielectric rod, but surprisingly, Fig. 2(a) shows the peak return occurred at $+5.9 \pm 0.4 \mathrm{~mm}, 9.0 \mathrm{~mm}$ delayed in range from the front surface. This offset is a consequence of the dielectric nature of the rod, which is transmissive to the millimeter wave probe, and the reflection from the concave back side of the rod produced a much stronger and directed return than the diverging return from its convex front surface. Indeed, the weak signal from the front of the dielectric rod is unseen because the range resolution is comparable to the rod diameter, and the strong signal from the back of the rod overwhelms it. However, the peak return appears to occur not from the back surface of the rod but behind it, a consequence of the permittivity of the dielectric. The peak of the delayed return $R_{\text {del }}$ is simply

$$
R_{\mathrm{del}}=d \sqrt{\epsilon_{r}} \text {. }
$$

Therefore, for a dielectric rod with permittivity $\epsilon_{r}=2$, $R_{\text {del }}=9 \mathrm{~mm}$ for a rod with diameter $d=6.4 \mathrm{~mm}$. Clearly the permittivity and shape of dielectric scatterers strongly affect the returns.

Both the SBR and MoM simulations agreed that a PEC rod will have a return at the front surface at a range of $-3.2 \mathrm{~mm}$. However, the SBR simulation for the dielectric rod, using the $\mathrm{XPATCH}$ setting for the dielectric function of bulk materials, inaccurately predicted the front peak to be at $-5.0 \mathrm{~mm}$, as shown in Fig. 2(a). Although it fairly accurately predicted the back peak at $+5.8 \mathrm{~mm}$, its strength was too low by more than $7 \mathrm{~dB}$. Consequently, SBR predicts that the dielectric rod returns will be (inaccurately) separated far enough so that returns from the front and back of the rod should be easily identified in the range domain, which they are not. It is important to note that the SBR simulation for the dielectric rod was accurate at $X$-band, so these inaccuracies only appear in this high frequency regime. By contrast, the MoM simulation [Fig. 2(a)] accurately predicted the measured return from the dielectric rod, placing the $9.0 \mathrm{~mm}$ delayed return at $+5.8 \mathrm{~mm}$ with a strength that matches the measured dielectric to within $1 \mathrm{~dB}$ and correctly does not resolve the reflection from the front surface.

More importantly, this analysis shows that ISAR techniques are able to distinguish metallic and dielectric scatterers, and this is accomplished even more clearly using SR algorithms. SR effectively involves expanding the spectral bandwidth of the ISAR data to improve radar range resolution. There are several SR algorithms, such as MUSIC, ESPIRIT [24], and Burg algorithms, the latter of which is used here. The Burg algorithm performs bandwidth extrapolation by using an iterative procedure to determine coefficients that minimize the sum of the forward and backward prediction error [25]. This algorithm was found to be computationally efficient and yielded a linearprediction filter that was stable. A zero-padded FFT smoothed the extrapolation, and the bandwidth could be extrapolated by as much as three times before significant extrapolation error 
and noise were introduced [25]. The tripled SR bandwidth of $105 \mathrm{GHz}$ produces W-band imagery with $1.4 \mathrm{~mm}$ range resolution, comparable to what could be achieved at G-band without SR. This can be seen by comparing the regular resolution RAI plot of the PEC rod in Fig. 2(b) with its SR RAI plot in Fig. 2(e), where the improved resolution of the return is consistently obtained from the front surface across all aspect angles.

This improvement can be also seen by comparing Figs. 2(a) and $2(\mathrm{~d})$, where the range resolution is three times narrower and the range returns appear sharper. Interestingly for the dielectric rod, applying SR to the initial measurement allows us to resolve both the weak front surface and strong, delayed rear surface returns. The dual return distinguishes the dielectric rod from PEC rods and their single front surface return. Notice that this double return signature is maintained for all aspect angles [Fig. 2(f)], and the dielectric rod optical thickness and location may be estimated by using these front and delayed return strengths and locations using Eq. (2) above. This important attribute of mmw ISAR with SR makes possible not only the identification of a target's location and shape but also its material composition, a capability rarely possible at traditional microwave frequencies where front and rear surface target reflections are usually too close to resolve [26].

\section{Complex Target}

RAI measurements of the complex target illustrated in Fig. 1 are shown in Fig. 3(a) and compared with predictions by both SBR and MoM methodologies in Figs. 3(b) and 3(c). The central rod has a slight tilt from vertical, which explains the minor variation of measured location with aspect angle, while the cylindrical rods offset from the center of rotation are distinguished by a smooth sinusoidal variation as they move toward or away from the transceiver while the target is rotated. By contrast, the PEC rectangular block has only strong specular returns every $90^{\circ}$ of rotation, specifically at $30^{\circ}, 120^{\circ}, 210^{\circ}$, and $300^{\circ}$, when each face is perpendicular to the transceiver. The edges of the rectangle scatter radiation too much to be identified.

In addition to the direct returns from the rods, labeled with an overlaid line of the same color as in Fig. 1, the measured data plotted in Fig. 3(a) exhibits extra returns past $50 \mathrm{~mm}$. These "artifact ghosts" are a consequence of the multipath interactions between the scatterers, producing the appearance of rangedelayed returns. Without simulations to interpret these returns, the direct and artifact returns could not be distinguished. Nevertheless, the image could be reconstructed because of the limited angular extent over which these artifacts appear. It is therefore noteworthy that the SBR simulation [Fig. 3(b)] does not predict these features, a clear indication of the deficiency of this technique to reproduce the data at high frequency. By contrast, the MoM simulation [Fig. 3(c)] exhibits significant delayed returns past $50 \mathrm{~mm}$, more than were seen in the experiment because of the expedient assumption that the target was composed of lossless PEC or dielectric rods instead of lossy aluminum and Teflon rods, exacerbated by the simulation of only a narrow vertical section of the target. Indeed, if the losses of the aluminum and Teflon rods were included instead of the lossless assumption
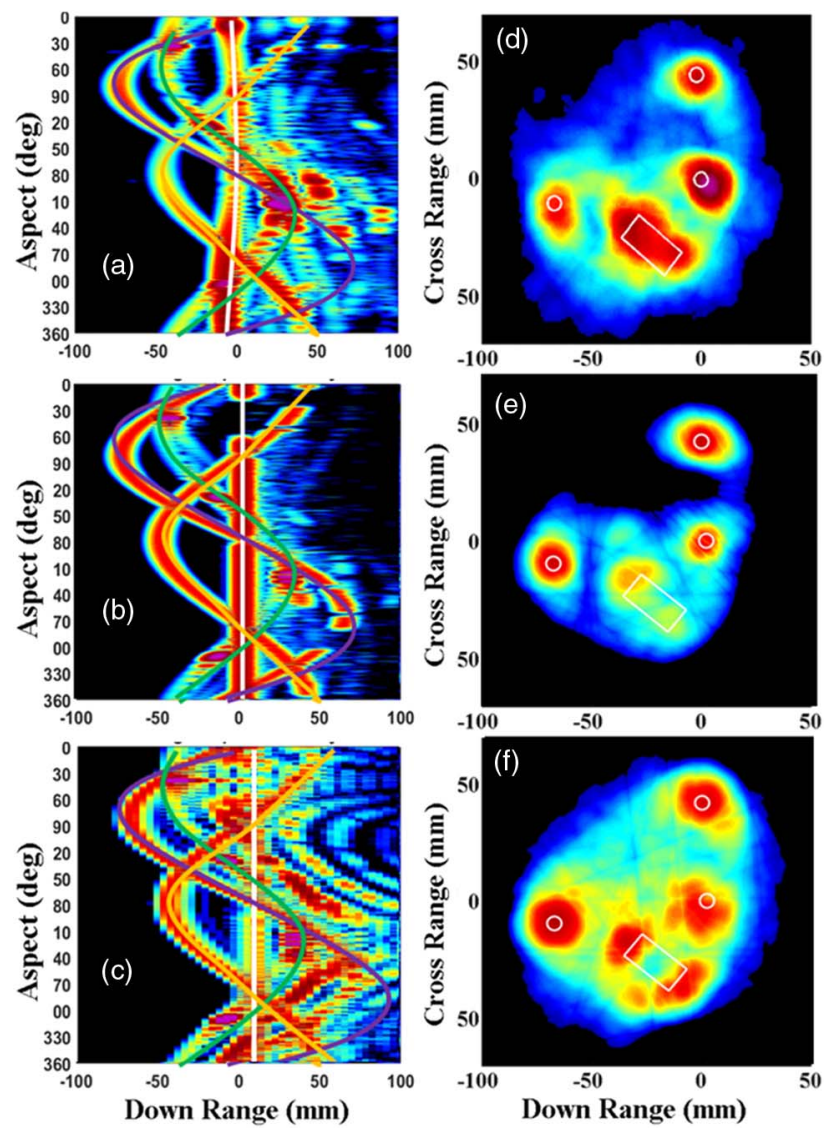

Fig. 3. RAI plots of the target for a $75-110 \mathrm{GHz}$ sweep with samples evenly spaced every $1^{\circ}$ across $360^{\circ}$ of rotation for the (a) measured data, (b) SBR simulation, and (c) MoM simulation of the complex, four-object target. As labeled in Fig. 1, the white line is object 1, the purple line is object 2, the orange line is object 3, and the green line is object 4 . Reconstructed ISAR images of the complex target using the Radon transform from $75-110 \mathrm{GHz}$ with 30 evenly spaced samples across $360^{\circ}$ of rotation for the (d) measured data, (e) SBR simulation, and (f) MoM simulation. The dynamic range from purple to black spans $45 \mathrm{~dB}$ in the RAI plots and $25 \mathrm{~dB}$ in the ISAR reconstructions.

used here for illustrative purposes, the agreement between the measurements and the MoM simulation would improve while the disagreement with the SBR simulation would worsen.

Although the PEC and dielectric rods could not be distinguished in the regular resolution data plotted in Fig. 3, this distinction is evident when the regular and SR plots of the complex target are compared in Figs. 4(a) and 4(e). The double return from the center rod in the SR plot of Fig. 4(e) indicates that it is a dielectric, while the rest of the rods are metallic and offset from the center of rotation. Note that if the dielectric center rod was replaced by two PEC point scatterers located where the front and back surfaces were, the signatures would sinusoidally oscillate in the RAI plot and vary in strength, just as these displaced PEC rods did, instead of displaying the constant dual returns for all aspects that is a characteristic of dielectrics.

SR also helps locate the rods more accurately, but at the cost of artificially increasing apparent noise throughout the ISAR 

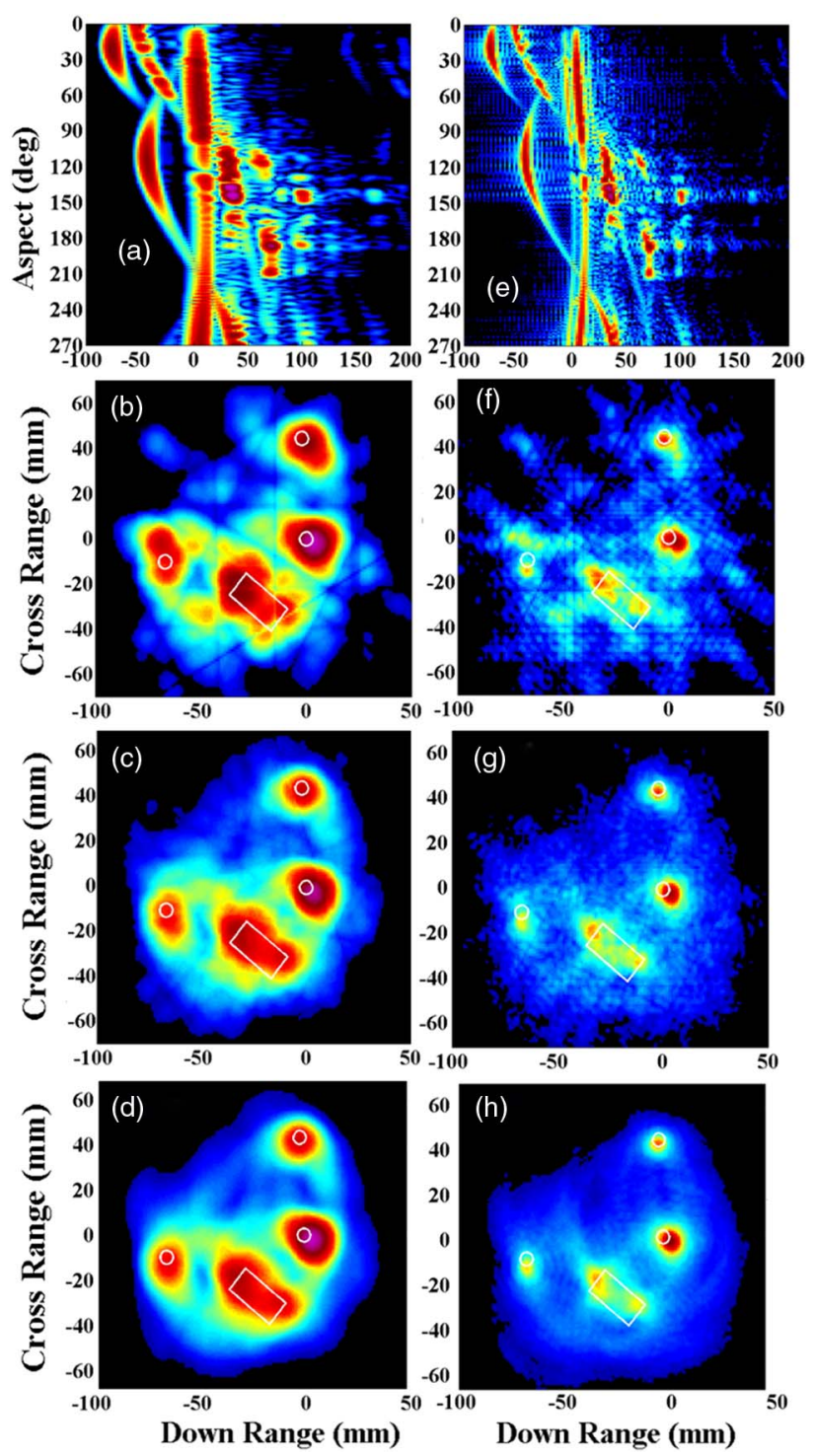

Fig. 4. (a) RAI plot from the range returns for a $75-110 \mathrm{GHz}$ sweep of linearly spaced samples measured across $360^{\circ}$ of rotation, and the associated Radon transform reconstructions for (b) 12, (c) 30, and (d) 360 evenly spaced angular samples. (e) RAI plot from the SR range returns for a $75-110 \mathrm{GHz}$ sweep of linearly spaced samples measured across $360^{\circ}$ of rotation, and the associated SR Radon transform reconstructions for (f) 12, (g) 30, and (h) 360 evenly spaced angular samples. The dynamic range from purple to black spans $45 \mathrm{~dB}$ in the RAI plots and $25 \mathrm{~dB}$ in the reconstructions.

image, as can be seen by comparing Figs. 4(a) and 4(e). The RAI plots indicate that one of the PEC rods, measured at the aspect angles of closest and farthest range $\left(30^{\circ}\right.$ and $\left.210^{\circ}\right)$, is located at $70 \mathrm{~mm}$ from the center of rotation with a range that oscillates sinusoidally with rotation. The other cylindrical PEC rod is located $50 \mathrm{~mm}$ from the center of rotation (maximum and minimum range angles of $120^{\circ}$ and $300^{\circ}$ ). The radial and angular locations of the rods measured with the RAI analysis agree with the locations measured with a ruler.

\section{ISAR TARGET RECONSTRUCTIONS}

\section{A. Complete Radon Reconstructions}

To reconstruct the locations of the scatterers in the complex object, we use a Radon transform on the RAI data, taking this series of one-dimensional (1D) datasets at particular aspect angles and integrating them over a 2D grid [27]. Radon transforms, which are routinely used to reconstruct images from computed tomography (CT) scans [28], were implemented using the filtered back projection algorithm. The Radon transform involves the creation of a wavefront image from $1 \mathrm{D}$ datasets acquired at a given angle, then rotating the image, repeating the acquisition, and combining intensities on a multidimensional grid to reconstruct the scene. Uncorrected, this would produce blurred images if no filtering (i.e., Hamming or Blackman-Harris window functions) was used on the datasets [17]. Of the two approaches for applying filters to improve the image, filters applied on each 1D dataset or a 2D filter applied on the final image, we used the former because it minimized the blurring effect.

As seen in Fig. 3(d), the Radon transform, using only 30 angular samples, recovers the locations of the isotropic rod scatters with an uncertainty of $1.0 \pm 0.5 \mathrm{~mm}$ in the $x-y$ coordinate system. In fact, the accuracy is better than the measured range and cross-range resolution of $\Delta r=4.3 \mathrm{~mm}$. The accuracy is also better than can be obtained from the RAI plots because the rod returns from all angles are included and the zeropadded FFT smoothed the data and improved the precision. Anisotropic scatterers like the rectangle were more difficult to locate [Fig. 3(d)] because they are visible over a smaller angular extent (i.e., strong specular reflection from the four faces, very weak scattering from the corners otherwise) and are thus much more vulnerable to shadowing by other scatterers.

Reconstructing the location of dielectric rods from reflected signals presents an interesting challenge for Radon transformbased reconstructions that are normally performed using transmitted (i.e., medical CT scans) or emitted (i.e., medical MRI scans) signals. Since the dominant return for dielectric scatterers could be delayed from the rear surface, the center of rotation could be off by a significant amount in location and return strength. For our dielectric rod, cylindrical symmetry allows the correct center location to be recovered (i.e., $(0 \mathrm{~mm}$, $0 \mathrm{~mm}$ ) in Fig. 3), but for more complex structures such as anisotropic scatterers, this offset must be recognized and characterized using an appropriate simulation to be compensated. For the measurement data with the dielectric rod at the center of rotation, the center was correct and the apparent width was the optical depth [Eq. (2)] rather than the physical depth.

The Radon transform of both the SBR and MoM simulations predicted the location and strength of the PEC rods accurately (Fig. 3). Both simulations also used the strong rear surface reflection to predict the location and strength of the dielectric rod correctly but, as noted above, the SBR underestimated the strength of the return and incorrectly resolved the reflection from the front surface. Reconstructing the PEC rectangle represented an interesting challenge for these techniques. The rectangle was difficult to recognize in the SBR simulation [Fig. 3(e)] with weak reconstructions that gave the impression of two isolated PEC scatterers instead. The rectangle was more 
obvious in the MoM simulation [Fig. 3(f)] because the walls were better circumscribed and more strongly defined while the center of the rectangle was void. This latter point is significant because the measured data [Fig. 3(d)] included a comparatively strong return from the entire volume of the rectangle, a consequence of imperfections not captured by the simulations (e.g., surface roughness and misalignment). Although the delayed returns from the multipath interference are obvious in the RAI plots [Figs. 3(a)-3(c)], the effects in the Radon transformed images [Figs. 3(d)-3(f)] are more subtle and manifest as a "halo" between the rods in the measured and MoM reconstructions, but are noticeably absent in the SBR reconstruction because of its failure to predict these artifacts. Given the large contribution from these multipath artifacts, the addition of simulated noise comparable to the measured noise insignificantly changes the quality of the simulated reconstructions from either model.

\section{B. Reduced Sample Radon Reconstructions}

An advantage to the Radon transform over traditional FFTbased ISAR algorithms [29] is that the Radon transform can generate images with a minimal number of angular samples. The Radon transform maintains the same range and crossrange resolution regardless of the number of samples, while traditional ISAR algorithms that use FFTs need a larger number of linearly spaced angular samples to provide high cross-range resolution [30]. Indeed, widely diverse angular sampling is desired to illuminate the entire target for the Radon transform [29], but are not completely necessary for other ISAR algorithms [29].

To explore how many samples are necessary to render an adequate reconstruction of our RAI data, the number of equally spaced angular samples used was increased from 4 to 360 . Figures $4(\mathrm{~b})-4(\mathrm{~d})$ illustrate this process with plotted reconstructions using 12,30 , and 360 samples, respectively. Reconstructions from reduced sampling produce idiosyncratic artifacts that are a consequence of the specific angles chosen for the specific target, as can be observed in the ancillary mottled features in Fig. 4(b). As the number of samples increase, those artifacts devolve into the multipath halo, as can be seen in the progression from Figs. 4(b) to 4(d). For a quantitative comparison of the reconstruction quality, the structural similarity (SSIM) method was used with the 360-sample reconstruction as the reference image [31]. As shown in Fig. 5, the quality and stability of the reconstructed image improved as the number of samples increased, exceeding 0.96 after 10 samples and 0.99 after 24 samples, after which the fidelity improved only marginally. Given that the sampling speed was determined by the rotation of the target, the fact that high-quality reconstructions could be achieved in 15-36 times fewer measurements represents a commensurate speed up in acquisition time. Depending on target complexity, an even greater acceleration may be possible if compressive sensing and adaptive sampling techniques are used [32].

Radon transforms of the SR RAI data were able to locate the center of the cylindrical scatterers with an uncertainty $<0.3 \mathrm{~mm}$ [Fig. 4(h)]. The ISAR image was then reconstructed with SR data for the same 12 and 30 samples above, as shown in Figs. 4(f) and 4(g), respectively. It is important to note that

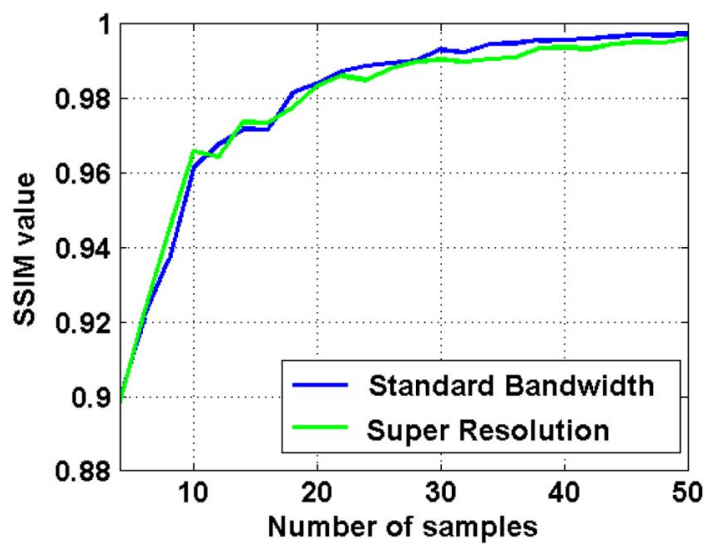

Fig. 5. SSIM values plotted as a function of the number of evenly spaced angular samples for the Radon reconstruction, as compared to the full Radon reconstruction with 360 samples, for standard and super resolution.

SR does not improve the image quality relative to its reference, as can be seen by comparing how the normal and SR SSIM values increase with increasing numbers of samples in Fig. 5. As in the normal resolution case, SSIM values of the 12 and 30 SR samples were 0.96 and 0.99 , respectively.

\section{CONCLUSIONS}

W-band ISAR images of several simple targets, generated through the use of Radon transforms, have been constructed and critically compared with predicted images using SBR and MoM simulations. As compared with the measured data, MoM simulations produce faithful reconstructions at these high frequencies and accurately predict the effects of multipath interference, while SBR simulations exhibit inferior reconstruction fidelity, create unphysical artifacts, and fail to predict measured artifacts. The need for accurate simulation tools to interpret the measured data is illustrated, a necessity that will only grow as target complexity increases. The data and simulations reveal clear signatures distinguishing metallic from dielectric scatterers. The delayed return from the dielectric rod proved that as higher bandwidth systems are used, the object structure and permittivity could potentially affect the location of the object as seen by the radar. The degradation of the image quality of Radon reconstructions with reduced data sets is found to be insignificant until fewer than 10 samples are taken, indicating the potential for reduced acquisition times and rapid reconstructions with an appropriately limited sampling strategy. SR techniques were able to achieve reconstructions with millimeter-scale resolution, indicating that rapid, high-fidelity, high-resolution image reconstructions using commercially available wide bandwidth W-band technology may obviate many of the advantages touted for higher-frequency, higher-bandwidth, higher-cost solutions at $\mathrm{THz}$ frequencies.

Funding. Missile S\&T program at the Army Aviation and Missile Research, Development, and Engineering Center (AMRDEC) through the Missile Defense Agency (HQ0147-14-C-7615). 
Acknowledgment. We thank Dane Phillips, Ruoyu Zhu, Daniel Marks, and David Brady for formative discussions about this project.

\section{REFERENCES}

1. J. Grajal, B. Mencia-Oliva, O. A. Yeste-Ojeda, A. F. GarciaFernandez, and G. Rubio-Cidre, "A prototype of high resolution ISAR imaging system at millimetre-wave band," in IEEE CIE International Conference on Radar (Radar), October 24-27, 2011, Vol. 1, pp. 551-554.

2. H. Stanko, D. Notel, A. Wahlen, J. Huck, F. Kloppel, R. Sommer, M. Hagelen, and $\mathrm{H}$. Essen, "Active and passive $\mathrm{mm}$-wave imaging for concealed weapon detection and surveillance," in 33rd International Conference on Infrared, Millimeter and Terahertz Waves (IRMMWTHz), September 15-19, 2008, pp. 1-2.

3. B. Mencia-Oliva, J. Grajal, and A. Badolato, "100-GHz FMCW radar front-end for ISAR and 3D imaging," in IEEE Radar Conference (RADAR), May 23-27, 2011, pp. 389-392.

4. H. Borrion, H. D. Griffiths, P. Tait, D. Money, and C. J. Baker, "Onedimensional model-based approach for ISAR imaging," in IGARSS '05: Proceedings of the IEEE International Geoscience and Remote Sensing Symposium, July 25-29, 2005, Vol. 1, p. 4.

5. A. B. del Campo, A. A. Lopez, B. P. Dorta Naranjo, J. G. Menoyo, D. R. Moran, and C. C. Duarte, "CWLFM millimeter-wave radar for ISAR imaging with range coverage," in IEEE International Radar Conference, May 9-12, 2005, pp. 933-938.

6. T. M. Goyette, J. C. Dickinson, J. Waldman, W. E. Nixon, and S. Carter, "Fully polarimetric W-band ISAR imagery of scale-model tactical targets using a 1.56-THz compact range," Proc. SPIE 4382 , 229-240 (2001).

7. G. P. Timms, M. L. Brothers, J. D. Bunton, J. W. Archer, G. C. Rosolen, Y. Li, A. D. Hellicar, J. Tello, and S. G. Hay, "Design and testing of an active 190-GHz millimeter-wave imager," J. Electron. Imaging 19, 043019 (2010).

8. B. Zhang, Y. Pi, and J. Li, "Terahertz imaging radar with inverse aperture synthesis techniques: system structure, signal processing, and experiment results," IEEE Sens. J. 15, 290-299 (2015).

9. S. Stanko, W. Johannes, R. Sommer, A. Wahlen, J. Wilcke, H. Essen, A. Tessmann, and I. Kallfass, "SAR with MIRANDA-millimeter wave radar using analog and new digital approach," in 8th European Radar Conference (EURAD) (IEEE, 2011), pp. 214-217.

10. M. Caris, S. Stanko, A. Wahlen, R. Sommer, J. Wilcke, N. Pohl, A. Leuther, and A. Tessmann, "Very high resolution radar at $300 \mathrm{GHz}$," in 11th European Radar Conference, Rome, Italy, 2014, pp. 494-496.

11. S. Bertl and J. Detlefsen, "Millimeter-wave imaging of dielectric objects using first order scattering models," in European Radar Conference (EuRAD), Amsterdam, The Netherlands, October 30-31, 2008, pp. 5-8.

12. A. V. Saylor, D. P. Barr, B. G. Woodruff, and L. T. Lowe, "W-band point-scatterer target models for HWIL simulations," Proc. SPIE 2741, 304-315 (1996).

13. Z. Li and T. J. Cui, "High frequency methods for simulation of high resolution imaging in terahertz regime," J. Infrared Millim. Terahertz Waves 31, 349-357 (2010).

14. C. J. Reddy, M. D. Deshpande, C. R. Cockrell, and F. B. Beck, "Fast RCS computation over a frequency band using method of moments in conjunction with asymptotic waveform evaluation technique," IEEE Trans. Antennas Propag. 46(8), 1229-1233 (1998).
15. J. Flodin, P. Kildal, and A. Kishk, "Moment method design of a large $\mathrm{S} / \mathrm{X}$ band corrugated horn," in Antennas and Propagation Society International Symposium, AP-S. Digest, July 21-26, 1996, Vol. 3, 2030-2033.

16. T. Ma, M.-S. Chen, X.-L. Wu, Q. Qi, Y. Liu, and M.-M. Hao, "Application of AWE technology combined with higher-order basis functions to broad band electromagnetic scattering problems," in International Conference on Microwave and Millimeter Wave Technology (ICMMT), May 5-8, 2012, Vol. 3, pp. 1-4.

17. F. J. Harris, "On the use of windows for harmonic analysis with the discrete Fourier transform," Proc. IEEE 66, 51-83 (1978).

18. M. Hazlett, D. J. Andersh, S. W. L. H. Ling, and C. L. Yu, "XPATCH: a high-frequency electromagnetic scattering prediction code using shooting and bouncing rays," Proc. SPIE 2469, 266 (1995).

19. Y. Tao, H. Lin, and H. Bao, "GPU-based shooting and bouncing ray method for fast RCS prediction," IEEE Trans. Antennas Propag. 58, 494-502 (2010).

20. C. C. Lu and W. C. Chew, "A coupled surface-volume integral equation approach for the calculation of electromagnetic scattering from composite metallic and material targets," IEEE Trans. Antennas Propag. 48, 1866-1868 (2000).

21. W. L. Stuzman and G. A. Theile, Antenna Theory and Design, 2nd ed. (Wiley, 1997).

22. Z. Kezhong, M. N. Vouvakis, and J.-F. Lee, "The adaptive cross approximation algorithm for accelerated method of moments computations of EMC problems," IEEE Trans. Electromagn. Compat. 47, 763-773 (2005).

23. C. Ronald, H. B. Wallace, and T. Blalock, "Numerical computation of the radar cross section of the ZSU-23-4," Army Research Lab Technical Report number, ARL-MR-430 (1999).

24. A. Quinquis, E. Radoi, and F. C. Totir, "Some radar imagery results using superresolution techniques," IEEE Trans. Antennas Propag. 52, 1230-1244 2004.

25. T. G. Moore, B. W. Zuemdorfer, and E. C. Burt, "Enhanced imagery using spectral-estimation-based techniques," Lincoln Lab. J. 10, 171187 (1997).

26. E. F. Knott, J. F. Shaeffer, and M. T. Tuley, Radar Cross Section (Artech House, 1993)

27. A. C. Kak and M. Slaney, Principles of Computerized Tomographic Imaging, Classics in Applied Mathematics (Society for Industrial and Applied Mathematics, 2001).

28. E. W. Weisstein, "Radon transform," from MathWorld-A Wolfram Web Resource, http://mathworld.wolfram.com/RadonTransform.html.

29. J. A. Noble and R. Narayanan, "Performance evaluation of the Radon transformation in limited angle radar tomography," http:// forms.gradsch.psu.edu/diversity/mcnair/mcnair_jrnl2012_14/files/ 2013-Noble.pdf.

30. A. Danylov, T. Goyette, J. Waldman, M. Coulombe, A. Gatesman, R. Giles, X. Qian, N. Chandrayan, S. Vangala, K. Termkoa, W. Goodhue, and W. Nixon, "Terahertz inverse synthetic aperture radar (ISAR) imaging with a quantum cascade laser transmitter," Opt. Express 18, 16264-16272 (2010).

31. Z. Wang, A. C. Bovik, H. R. Sheikh, and E. P. Simoncelli, "Image quality assessment: from error visibility to structural similarity," IEEE Trans. Image Process. 13, 600-612 (2004).

32. R. Zhu, J. T. Richard, D. J. Brady, D. L. Marks, and H. O. Everitt, "Compressive sensing and adaptive sampling applied to millimeter wave inverse synthetic aperture imaging," Opt. Express 25, 2270 2284 (2017) 